\title{
FIELDS IN WHICH VARIETIES HAVE RATIONAL POINTS: A NOTE ON A PROBLEM OF AX
}

\author{
NEWCOMB GREENLEAF
}

If $k$ is a field, let $G(k)$ denote the Galois group over $k$ of the algebraic closure of $k$. In [1] Ax proved the following result:

Let $k$ be a perfect field such that $G(k)$ is abelian, and every (absolutely irreducible) variety defined over $k$ has a $k$-rational point. Then $G(k)$ is pro-cyclic.

He asked [1, Problem 1] if the assumption that $G(k)$ is abelian can be removed. In this note we construct an example to show that this assumption is necessary. Recall that if $X$ is a variety defined over $k$, then $k(X)$ is a regular extension of $k$ [2]. Further $X$ contains a $k(X)$-rational point (in fact a $k(X)$-rational point which is generic over $k$ ).

[ADDED IN PROOF. A negative answer to Probelm 1 of [1] has also been given by Moshe Jarden (Rational points on algebraic varieties over large number fields, Bull. Amer. Math. Soc. 75 (1969), 603-606).]

If $E$ and $F$ are regular extensions of $k$, then the free composite of $E$ and $F$ over $k$ is again a regular extension of $k$. Let $\left\{E_{\alpha}\right\}$ be any set of regular extensions of $k$. By an easy transfinite induction we can construct the free composite of $\left\{E_{\alpha}\right\}$ over $k$, and it will again be a regular extension of $k$.

Now let $\left\{E_{\alpha}\right\}$ be a complete set of nonisomorphic finitely generated regular extensions of $k$, and let $E(k)$ denote their free composite. Then $E(k)$ is a regular extension of $k$ and every variety $X$, defined over $k$, has an $E(k)$-rational point. Set $E^{n}(k)=E\left(E^{n-1}(k)\right)$ and $E^{\infty}(k)=\bigcup_{n=1}^{\infty} E^{n}(k)$.

If $X$ is a variety defined over $E^{\infty}(k)$, then $X$ is actually defined over $E^{n}(k)$ for some $n$, and hence has an $E^{n+1}(k)$-rational point. Since $k$ is algebraically closed in $E^{\infty}(k)$, the map $G\left(E^{\infty}(k)\right) \rightarrow G(k)$ is surjective, so $G(k)$ nonabelian implies $G\left(E^{\infty}(k)\right)$ nonabelian. Finally if $k$ is of characteristic zero, then $E^{\infty}(k)$ is perfect. Hence if we take, say $k=Q$, we get the desired example.

Remark. In an oral communication, S. Garfunkel has pointed out to Ax that Problem 5 of [1] has an affirmative solution, i.e. that the theory of the rings $Z / m$ is decidable. This follows from Corollaries

Received by the editors January 20, 1969 and, in revised form, April 23, 1969. AMS 1970 subject classifications. Primary 02H15, 14G99, 12L10. 
1 and 2 to Theorem 17 of [1], combined with Theorem 7.9 from reference 10 of [1].

\section{REFERENCES}

1. J. Ax, The elementary theory of finite fields, Ann. of Math. (2) 88 (1968), 239271. MR 37 \#5187.

2. S. Lang, Introduction to algebraic geometry, Interscience, New York, 1958. MR 20 \#7021.

UNIVERSITY OF ROCHESTER, ROCHESTER, NEW YORK 14627

University of California, Berkeley, California 94720 